\title{
Equivalence of nonlinear systems to triangular form: the singular case
}

\author{
Sergej Čelikovský ${ }^{\mathrm{a}, *}$, Henk Nijmeijer $^{\mathrm{b}}$ \\ anstitute of Information Theory and Automation, Academy of Sciences of the Czech Republic, \\ P.O. Box 18, 18208 Prague, Czech Republic \\ ${ }^{\mathrm{b}}$ Department of Applied Mathematics, University of Twente, P.O. Box 217, 7500 AE Enschede. The Netherlands
}

Received 20 June 1995; revised 27 October 1995

\begin{abstract}
The problem of state equivalence of a given nonlinear system to a triangular form is considered here. The solution of this problem has been known for the regular case, i.e. when there exists a certain nested sequence of regular and involutive distributions. It is also known that in this case the corresponding system is linearizable using a smooth coordinate change and static state feedbark. This paper deals with the singular case, i.e. when the nested sequence of involutive distributions of the system contains singular distributions. Special attention is paid to the so-called bijective triangular form. Geometric, coordinates-free criteria for the solution of the above problem as well as constructive, verifiable procedures are given. These results are used to obtain a result in the nonsmooth stabilization problem.
\end{abstract}

Keywords: Nonlinear systems; Triangular form; Nonsmooth stabilization

AMS Classification: 93C10; 93B10; 93D15

\section{Introduction}

We consider a single-input nonlinear system

$\dot{x}=f(x)+u g(x), \quad x \in \mathbb{R}^{n}, u \in \mathbb{R}$,

where $f, g$ are smooth vector fields. We are interested in studying the local structure of this system in a neighbourhood of an equilibrium point, that is for simplicity assumed to be the origin, i.e. $f(0)=0$. Our aim is to find coordinate-free conditions for local equivalence

\footnotetext{
*Corresponding author. E-mail: celikovs@utia.cas.cz. This work was performed while the author was visiting the Department of Applied Mathematics of the University of Twente as a research fellow supported in part by the Dutch Network on Systems and Control Theory and the Department of Applied Mathematics. The author is also partially supported by the Grant Agency of the Czech Republic through Research Grant No. 102/94/0053.
}

of (1) to a triangular form $(T F)$

$$
\begin{aligned}
\dot{x}_{1} & =f_{1}\left(x_{1}, x_{2}\right), \\
\dot{x}_{2} & =f_{2}\left(x_{1}, x_{2}, x_{3}\right), \\
& \vdots \\
\dot{x}_{n-1} & =f_{n-1}\left(x_{1}, x_{2}, \ldots, x_{n}\right), \\
\dot{x}_{n} & =f_{n}\left(x_{1}, x_{2}, \ldots, x_{n}\right)+u g_{n}(x), \\
g_{n}(x) & \neq 0, \frac{\partial f_{i}}{\partial x_{i+1}}(x) \neq 0, \quad i=1,2, \ldots, n-1 \\
\forall x & \in \mathscr{N} \subset \mathscr{N}_{0}, \overline{\mathcal{N}}=\mathscr{N}_{0},
\end{aligned}
$$

$\mathscr{N}_{0}$ being a neighbourhood of the origin and $\mathscr{N}$ an open dense subset that may not contain the origin.

Here the equivalence we use is that of selecting appropriate local coordinates around the origin. Beginning with [9], triangular form nonlinear systems 
have received a lot of attention. One reason that the triangular structure has been investigated is the close connection between "triangularizability" and feedback linearization (see $[8,10]$ ). Another motivation forms the study of feedback stabilization of systems of the form (1), see e.g. [17-19], where for some specific TF-systems a stabilization theory has been developed, or [6]. In both cases a particular role is played by TF-systems in which each of the components $f_{i}, i=$ $1, \ldots, n-1$, is a local bijection in its last argument and $g_{n}(0) \neq 0$ (see for a precise definition later on); in this case we refer to a bijective triangular form $(B T F)$. A sufficient condition for the above bijectivity property is that $g_{n}(x) \neq 0,\left(\partial f_{i} / \partial x_{i+1}\right)(0) \neq 0, \forall i=$ $1, \ldots, n-1$; this case is referred as the regular case.

The problem of the equivalence to the TF was completely solved in the regular case by Jakubczyk and Respondek in [8] where the solution gives conditions for exact feedback linearization of (1). For a detailed survey of the regular case see also the monographs $[10,7]$. Nevertheless, only few results are known for the singular case (i.e. the case that is not regular) and these results usually give special triangular forms, see e.g. [12] and further references in there. We give here necessary and sufficient conditions for the equivalence of (1) to the triangular form and also necessary and sufficient conditions for this form to be bijective. The basic result is that these conditions also cover the singular case and are given in a coordinate-free form.

Our study of the problem of the equivalence to singular triangular form was partially motivated by its discrete-time version investigated in [14] in connection with the nonsmooth feedback stabilization and linearization of discrete-time systems. However, that paper does not study coordinates-free conditions for the TF to be BTF.

The paper is organized as follows. Section 2 introduces notation and gives necessary definitions together with some preliminary results. The main results are formulated and illustrated in Section 3 while their proofs are provided in Section 4. Finally, application to the nonsmooth stabilization problem is considered in Section 5. We conclude the paper by giving some remarks and outlooks in Section 6.

\section{Definitions and preliminary results}

First, we introduce further necessary notation and terminology. We say that the TF-system (2) is locally around the origin in bijective triangular form $(B T F)$ if $g_{n}(0) \neq 0$ and there exists a positive real number $\varepsilon$ such that $\forall i=1, \ldots, n-1, \forall x_{k} \in(-\varepsilon, \varepsilon), k=1, \ldots, i$, the following condition holds:

$$
\begin{aligned}
& f_{i}\left(x_{1}, x_{2}, \ldots, x_{i}, \cdot\right):(-\varepsilon, \varepsilon) \\
& \quad \mapsto f_{i}\left(x_{1}, x_{2}, \ldots, x_{i},(-\varepsilon, \varepsilon)\right) \text { is a bijection. }{ }^{1}
\end{aligned}
$$

Notice that if the BTF for the single-input affine system (1) exists, one has after a suitable (local) coordinate change $g_{n} \equiv 1$.

Recall that by the above mentioned regular case we understand the case when in (4) all bijections are local diffeomorphisms, i.e. $\forall i\left(\partial f_{i} / \partial x_{i+1}\right)(0) \neq 0$. By the singular case we understand the case which is not regular, i.e. $\left(\hat{\partial} f_{i} / \partial x_{i+1}\right)(0)=0$ for some $i$.

We use throughout the paper standard differentialgeometric notions and notation (see e.g. $[10,7]$ ). Since our results will be local, we restrict ourselves to systems evolving on $\mathbb{R}^{n}$. Besides the frequently used regular distributions we consider singular distributions: a distribution $\Delta(x)$ is for all $x \in \mathbb{R}^{n}$ a subspace of $T_{x} \mathbb{R}^{n}$ with dimension possibly varying with respect to $x$. A distribution is called smooth (analytic) if it is spanned by a (possibly infinite) set of smooth (analytic) vector fields. We call a set of smooth vector fields $\mathscr{F}$ to be locally finitely generated (LFG) if, locally around each $x \in \mathbb{R}^{n}$, there exists a finite set of vector fields $\mathscr{T}$ such that any vector field from $\mathscr{F}$ may be expressed as $\sum_{k=1}^{s} \alpha_{k}(x) \tau_{k}(x), \tau_{k} \in \mathscr{T}, \alpha_{k} \in C^{\infty}\left(\mathbb{R}^{n}\right), s<\infty$. A set of vector fields $\mathscr{T}$ is called involutive if it is closed with respect to taking Lie bracket, i.e. $\forall \tau_{1}, \tau_{2} \in$ $\mathscr{T}\left[\tau_{1}, \tau_{2}\right] \in \mathscr{T}$. Notice that a noninvolutive set of vector fields may span an involutive singular distribution $\Delta$, since the last property means that $\forall \tau_{1}, \tau_{2} \in \mathscr{T}$ such that $\tau_{1,2}(x) \in \Delta(x), \forall x$, we have that $\left[\tau_{1}, \tau_{2}\right](x) \in$ $\Delta(x), \forall x$. Take for example $\mathscr{T}=\left\{(0,1)^{\prime},\left(x_{2}^{2}, 0\right)^{\prime}\right\}$ then $\left[(0,1)^{\prime},\left(x_{2}^{2}, 0\right)^{\prime}\right]=\left(2 x_{2}, 0\right)^{\prime} \notin \mathscr{T}$, i.e. $\mathscr{T}$ is not an involutive set of vector fields. On the other hand, the distribution $\Delta(x)=\operatorname{span}\left\{(0,1)^{\prime},\left(x_{2}^{2}, 0\right)^{\prime}\right\}$ is an involutive singular distribution on $\mathbb{R}^{2}$ since for all $x \in$ $\mathbb{R}^{2}\left[(0,1)^{\prime},\left(x_{2}^{2}, 0\right)^{\prime}\right]=\left(2 x_{2}, 0\right)^{\prime} \in \Delta(x)$.

For a given set of vector fields $\mathscr{T}$ we denote by $[\mathscr{T}]$ its involutive closure, i.e. the smallest (with respect to the inclusion ) involutive set of vector fields containing it. Obviously, $\mathscr{T}$ is involutive if and only if $[\mathscr{T}]=$ $\mathscr{T}$. Recall that in the analytic case a finitely generated set of vector fields has always a finitely generated

\footnotetext{
${ }^{1}$ In fact, due to the assumption of smoothness this mapping is a homeomorphism.
} 
involutive closure; in the $C^{\infty}$ case this may not be true (see [7] for further details).

To simplify and shorten statements we often allow a slight abuse of notations: where no confusion arises $x$ may stand for several different coordinates systems.

Throughout the paper we will use the following nested sequence of distributions generated by the system (1),

$$
\begin{gathered}
\Delta_{i}(x)=\operatorname{span}\left\{g(x), \operatorname{ad}_{f} g(x), \ldots, \operatorname{ad}_{f}^{i} g(x)\right\}, \\
i=0, \ldots, n-1,
\end{gathered}
$$

and define the matrices

$$
\begin{gathered}
\mathscr{M}_{i}(x)=\left[g(x)\left|\operatorname{ad}_{f} g(x)\right| \ldots \mid \operatorname{ad}_{f}^{i} g(x)\right], \\
i=0, \ldots, n-1 .
\end{gathered}
$$

The following lemma is important in the paper (see $[8,11])$.

Lemma 1. Consider a nested sequence of smooth involutive constant dimensional distributions

$\Delta_{0} \subset \Delta_{1} \subset \cdots \Delta_{k}, \quad k \geqslant 0$.

Then locally there exist smooth coordinates $x$ such that

$$
\begin{aligned}
& \Delta_{i}(x)=\operatorname{span}\left\{\frac{\partial}{\partial x_{1}}, \ldots, \frac{\partial}{\partial x_{l_{i}}}\right\}, \\
& l_{i}=\operatorname{dim} \Delta_{i}, i=0,1, \ldots, k .
\end{aligned}
$$

The following theorem is an adaptation of the wellknown result on exact feedback linearization (see $[8,10]$ ) and completely solves the problem of state equivalence to the regular triangular form.

Theorem 1. The foliowing statements are equivalent:

1. There exist locally around the origin coordinates taking system (1) into the regular triangular form (2)-(4).

2. For all $i=0, \ldots, n-1$, the distribution $\Delta_{i}$ given by (5) is involutive and regular around the origin with $\operatorname{dim} \Delta_{i}=i+1$.

3. The original nonlinear system is locally feedback linearizable using a smooth coordinate change and a smooth static state feedback.

Furthermore, in analogy with Theorem 2.1.7 of [7], for a nested sequence of smooth singular distributions (7) with each $\Delta_{i}$ being LFG, we have that each distribution $\Delta_{i}$ has the maximal integral manifold property and the corresponding submanifolds at each point are also nested.

First, we formulate and prove a continuous-time analogue of Corollary 4.6 of [14]

Theorem 2. Consider the smooth system (1). The following statements are equivalent.

1. There exist local coordinates around the origin taking system (1) into the TF (2)-(3).

2. There exists a neighbourhood $\mathscr{N}_{0}$ of the origin and an open subset $\mathscr{N}$ with $\overline{\mathcal{N}}=\mathscr{N}_{0}$ such that:

(a) the distributions (5) are constant dimensional and involutive on $\mathscr{N}$ with $\operatorname{dim} A_{n-1}(x)=n \forall x \in \mathscr{N}$,

(b) on $\mathscr{N}_{0}$ there exists a nested sequence of regular distributions $\left\{\bar{\Delta}_{i}\right\}_{i=1}^{n}$ such that

$\bar{\Delta}_{i}(x)=\Delta_{i}(x) \quad \forall x \in \mathscr{N}, i=0,1, \ldots, n-1$.

Before proving the above theorem, we give an equivalent formulation of 2.(b) that indicates how to check this condition for a particular system.

Corollary 1. Suppose that Theorem 2.2.(a) is valid. Then Theorem 2.2.(b) is equivalent to:

$2\left(b^{*}\right)$ For all $i=0,1, \ldots, n-1$ there exist an $(n \times(i+1))$ matrix $\overline{\mathscr{M}}_{i}(x)$ and an $((i+1) \times(i+1))$ matrix $v_{i}(x)$ such that

$\forall x \in \mathcal{N}_{0}: \mathscr{M}_{i}(x)=\overline{\mathscr{M}}_{i}(x) v_{i}(x)$,

where the matrix $\mathscr{M}_{i}(x)$ given by (6) has full rank on $\mathscr{N}$, the matrix $\bar{M}_{i}(x)$ has full rank on $\mathscr{N}_{0}$ and the square matrix $v_{i}(x)$ has full rank on $\mathscr{N}$. Simply said, the singularity can be factored out from the matrix $\mathscr{M}_{i}(x)$. Moreover, all factorizations (10) may be selected in such a way that all $v_{i}(x)$ are lower antitriangular ${ }^{2}$ and $v_{i_{1}}(x)$ is a submatrix of $v_{i_{2}}(x)$ for all $i_{1}<i_{2}$.

Proof of Corollary 1. Suppose Theorem 2.2.(b) and 2.2.(a) is valid, then by Theorem 2 there exist triangular coordinates and in these coordinates straightforward computations give $\mathscr{M}_{i}=\left[0_{(i+1) \times(n-i-1)} \mid v_{i}(x)\right]^{\prime}$, where $v_{i}(x)$ is an $(i+1) \times(i+1)$ lower anti-triangular matrix with $v_{i_{1}}(x)$ being a submatrix of $v_{i_{2}}(x)$ for all $i_{1}<i_{2}$. The factorization (10) is then obvious, namely, take $\overline{\mathscr{M}}_{i}=\left[0_{(i+1) \times(n-i-1)} \mid I_{(i+1) \times(i+1)}\right]^{\prime}$. The geometric meaning of $(10)$ is the following: the columns of both $\overline{\mathscr{M}}_{i}$ and $\mathscr{M}_{i}$ are vector fields while elements of $v_{i}$ are $C^{\infty}$ functions needed to express any vector field

\footnotetext{
${ }^{2}$ That is, all elements above its anti-diagonal are equal to zero.
} 
- column of $\mathscr{M}_{i}$ - via those of $\overline{\mathscr{M}}_{i}$. As an immediate consequence we have that if the lower anti-triangular factorization (10) (having the submatrix property of $v_{i}$ 's) exists in some particular coordinates, then it exists in all coordinates, i.e. Corollary 1.2.( $\left.\mathbf{b}^{*}\right)$ is valid.

Conversely, if Theorem 2.2.(a) is valid together with Corollary 1.2.( $\left.\mathrm{b}^{*}\right)$ then the distributions $\bar{\Delta}_{i}$ in Theorem 2.2.(b) obviously exist, namely, they are spanned by the columns of $\overline{\mathscr{M}}_{i}$.

Remark 1 (Important computational observation). The existence of factorizations (10) with lower antitriangular matrices $v_{i}$ may be checked in the following constructive way (recursively with $i=0, \ldots, n-1$ ):

1. For $i=0$ we have to find a smooth scalar function $v_{0}$ such that $g / v_{0}$ is smooth and nonzero at the origin. If such a $v_{0}$ does not exist, we conclude that the factorization (10) does not exist as well. Otherwise, we have the lower anti-triangular factorization (10) for $i=0$, namely: $g(x)=\left(g(x) / v_{0}(x)\right) v_{0}(x)$.

2. Recursive step. Suppose we have already obtained lower anti-triangular factorizations for $i=$ $0, \ldots, k, 0 \leqslant k \leqslant n-1$. Full rank of $\overline{\mathscr{M}}_{k}(x)$ implies that $R_{k}(x) \overline{\mathscr{M}}_{k}(x)=\left[0_{(k+1) \times(n-k-1)} \mid D_{k}(x)\right]^{\prime}$ where $D_{k}(x)$ is a $((k+1) \times(k+1))$ nonsingular diagonal matrix and $R_{k}(x)$ is an $(n \times n)$ nonsingular matrix representing row operations needed to take $\overline{\mathscr{M}}_{k}(x)$ into the form $\left[0_{(k+1) \times(n-k-1)} \mid D_{k}(x)\right]^{\prime}$. Let $R_{k}(x) \operatorname{ad}_{f}^{k+1} g=\left[\alpha_{1}(x), \ldots, \alpha_{n}(x)\right]^{\prime}$ and $\alpha^{l}=$ $\left[\alpha_{1}, \ldots, \alpha_{n-k-1}\right]^{\prime}, \quad x^{2}=\left[\alpha_{n-k}, \ldots, \alpha_{n}\right]^{\prime}$. We have $R_{k}(x) \mathscr{M}_{k+1}(x)=\left[R_{k}(x) \mathscr{M}_{k}(x) \mid R_{k}(x) \mathrm{ad}_{f}^{k+1} g\right]$, i.e. using (10) and the notation just introduced:

$$
\begin{array}{r}
R_{k}(x) \mathscr{M}_{k+1}(x)=\left[R_{k}(x) \overline{\mathscr{M}}_{k}(x) v_{k}(x) \mid\left[\alpha^{1}, \alpha^{2}\right]^{\prime}\right] \\
\quad=\left[\left[0_{(k+1) \times(n-k-1)} \mid D_{k}(x)\right]^{\prime} v_{k}(x) \mid\left[\alpha^{1}, \alpha^{2}\right]^{\prime}\right] .
\end{array}
$$

If there exists a smooth function $\beta_{k+1}(x)$ such that $\alpha^{1}(x) / \beta_{k+1}(x)$ is smooth and nonzero at the origin, then (10) is valid also for $i=k+1$ with

$$
\begin{aligned}
& \overline{\mathscr{H}}_{k+1}=R_{k}^{-1}\left[\begin{array}{cc}
\alpha^{1} / \beta_{k+1} & 0 \\
0 & D_{k}
\end{array}\right], \\
& v_{k+1}=\left[\begin{array}{cc}
0 & \beta_{k+1} \\
v_{k} & D_{k}^{-1} \alpha^{2}
\end{array}\right] .
\end{aligned}
$$

Finally, it may be shown that if no $\beta_{k+1}$ exists for which $\alpha^{1}(x) / \beta_{k+1}(x)$ is smooth and nonzero at the origin, then the factorization (10) does not exist as well. The recursive step is complete.

Notice that the matrices $R_{0}, \ldots, R_{n-1}$ may be also easily computed recursively: if $R_{k}(x) \overline{\mathscr{M}}_{k}(x)=$ $\left[0_{(k+1) \times(n-k-1)} \mid D_{k}(x)\right]^{\prime}$, then $R_{k+1}=\tilde{P}^{n-k-1} \tilde{R}_{k+1} R_{k}$ where $\tilde{R}_{k+1}$ is such that $\tilde{R}_{k+1} \alpha^{1}=\left[0, \ldots, 0, \beta_{k+1} d_{k+1}\right]^{\prime}$, $d_{k+1}(0) \neq 0$ (recall that $\alpha^{1} / \beta_{k+1}$ is smooth and nonzero at the origin), and $\tilde{P}^{n-k-1}=\left(\tilde{p}_{i, j}\right), i, j=$ $1, \ldots, n$, is the permutation matrix with zero entries except $\tilde{p}_{1,1}=\cdots=\tilde{p}_{n-k-2, n-k-2}=\tilde{p}_{n, n-k-1}=$ $\tilde{p}_{n-k-1, n-k}=\cdots=\tilde{p}_{n-1, n}=1$. Moreover, the only matrices to be inverted are either diagonal or represent row operations.

In other words, only two simple tools are necessary either to obtain lower anti-triangular factorizations (10) or to exclude their existence, namely: finding a common scalar factor of a column vector and row operations.

Proof of Theorem 2. $1 \Rightarrow 2$. Suppose system (1) is in $\mathrm{TF}(2)$. Introducing the following notations,

$$
\begin{aligned}
& e_{i}=\left(0, \ldots, 0,1_{i \text { th }}, 0, \ldots, 0\right)^{\prime}, \quad i=1, \ldots, n, \\
& d_{1}(x)=g_{n}(x), \quad d_{i+1}(x)=d_{i}(x) \frac{\partial f_{n-i}}{\partial x_{n-i+1}}(x), \\
& \quad i=1, \ldots, n-1, x \in \mathscr{F}_{0},
\end{aligned}
$$

we have, after straightforward computations, for all $x \in \mathcal{A}$

$$
\begin{aligned}
& \Delta_{0}(x)=\operatorname{span}\left\{\left(0, \ldots, 0, d_{1}(x)\right)^{\prime}\right\}, \\
& \Delta_{i+1}(x)=\operatorname{span}\left\{\Delta_{i}(x), e_{n-i-1} d_{i+2}(x)\right\}, \\
& \quad i=0, \ldots, n-2,
\end{aligned}
$$

so that, obviously, $\Delta_{i}(x)$ has the property 2 .(a) while $\bar{\Delta}_{i}(x)=\operatorname{span}\left\{e_{n-i}, \ldots, e_{n}\right\}, i=0, \ldots, n-1$, are the distributions required by 2.(b).

$2 \Rightarrow 1$. Applying Lemma 1 to the nested sequence of regular involutive distributions $\bar{J}_{i}(x)$ having property (9) we obtain their flat coordinates $x$ for which $\bar{\Delta}_{i}(x)=\operatorname{span}\left\{\partial / \partial x_{n-i}, \ldots, \partial / \partial x_{n}\right\}$ and therefore due to 2.(b)

$\frac{\partial f_{i}}{\partial x_{j}}(x)=0 \quad \forall x \in \mathscr{N}, \forall i, j \in\{1, \ldots, n\}$,

such that $j \geqslant i+2$.

The validity of the last relations extends by a limit argument to all $x \in \mathscr{N}_{0}=\overline{\mathcal{N}}$, i.e. the system is in the TF (2). Now, condition 2.(a) guarantees that this TF has property (3).

The condition 2.(b) of the previous theorem is actually crucial for the equivalence to the TF in the case 
of singular distributions (5). One can easily check its validity for a particular sequence of distributions. It can be violated in two different ways. Perhaps, it is not surprising that for smooth nonanalytic systems one may easily construct an example violating Theorem 2.2.(b) (using the familiar function $\phi(x)=$ $\exp (-1 / x), x>0, \phi(x)=0, x \leqslant 0)$. A more interesting fact is that even an analytic system having property 2.2.(a) of Theorem 2 need not comply with Theorem 2.2.(b), as is illustrated by the following example.

Example 1. Consider the three-dimensional singleinput system

$\dot{x}_{1}=x_{2}+x_{1} x_{3}, \quad \dot{x}_{2}=2 x_{1}+2 x_{2} x_{3}, \quad \dot{x}_{3}=u$.

Here Theorem 2.2.(a) is obviously valid since

$$
\begin{aligned}
& \Delta_{0}(x)=\operatorname{span}\left\{(0,0,1)^{\prime}\right\}, \\
& \Delta_{1}(x)=\operatorname{span}\left\{(0,0,1)^{\prime},\left(x_{1}, 2 x_{2}, 0\right)^{\prime}\right\}, \\
& \Delta_{2}(x)=\operatorname{span}\left\{(0,0,1)^{\prime},\left(x_{1}, 2 x_{2}, 0\right)^{\prime},\left(-x_{2}, 2 x_{1}, 0\right)^{\prime}\right\},
\end{aligned}
$$

but there does not exist a regular distribution $\bar{\Delta}_{1}(x)$ with property (9). Actually, there does not exist smooth function $\beta(x)$ such that $\left(x_{1}, 2 x_{2}, 0\right)^{\prime} / \beta(x)$ is nonzero and smooth at the origin, i.e. by Remark 1 Theorem 2.2.(b) is not valid and the investigated system is not equivalent to TF. Alternatively, the situation allows the following clear geometrical description. Observe that the distribution $\Delta_{1}(x)$ has the maximal integral manifold property (using Theorem 2.1.7 of [7]) and the corresponding manifolds have a very peculiar topological structure around the origin: through the point $\left(x_{1}^{0}, x_{2}^{0}, x_{3}^{0}\right)^{\prime}$ the maximal integral manifold is given as

$$
\begin{gathered}
\left\{x=\left(x_{1}, x_{2}, x_{3}\right)^{\prime} \in \mathbb{P}^{3} \mid x_{1}=x_{1}^{0} \exp \left(\tau_{1}\right),\right. \\
\left.x_{2}=x_{2}^{0} \exp \left(2 \tau_{1}\right), x_{3}=\tau_{2}, \tau_{1,2} \in \mathbb{R}\right\},
\end{gathered}
$$

i.e. there is a bundle of parabolic surfaces crossing each other at the line $x_{1}=x_{2}=0$. Each such parabolic surface is composed from two two-dimensional maximal integral manifolds and from the line $x_{1}=x_{2}=0$, itself being a one-dirnensional maximal integral manifold. Since the distribution $\bar{\Delta}_{1}(x)$ should be regular and involutive with property (9), its integral manifolds should be every'where two-dimensional and they should be everywhere except the line $x_{1}=x_{2}=0$ coincide with the above-described maximal integral manifolds. The last property is an obvious contradiction, i.e. Theorem 2.2.(b) is not valid.

Before further investigating the existence of the BTF we give an equivalent formulation of Theorem 2 that will be used in the sequel. Moreover, it provides a rather clear geometric insight.

Theorem 2*. Consider the smooth system (1). The following statements are equivalent.

1. There exist local coordinates around the origin taking system (1) into the TF (2)-(3).

2. On a neighbourhood $\mathcal{N}_{0}$ of the origin there exist smooth exact one-forms $\psi_{i}, i=0,1, \ldots, n-1$, such that for all $i=0,1, \ldots, n-1$ :

(a) $\left\langle\psi_{i}, \operatorname{ad}_{f}^{i} g\right\rangle \neq 0$ for all $x \in \mathscr{N}, \mathscr{N}$ open, $\overline{\mathcal{N}}=\mathscr{N}_{0}$

(b) dim span $\left\{\psi_{0}, \ldots, \psi_{i}\right\}=i+1,\left\langle\psi_{i}, \operatorname{ad}_{f}^{j} g\right\rangle=0$, for all $j<i$ and for all $x \in \mathscr{N}_{0}$.

Proof. $1 \Rightarrow 2$. This implication is obvious: take triangular coordinates $x$ for the system (1), then $\psi_{i}=$ $\mathrm{d} x_{n-i}, i=0, \ldots, n-1$, are exact forms satisfying 2 .

$2 \Rightarrow 1$. One can easily see (using a dual form of the Frobenius theorem - see e.g. [10]) that the distributions $A_{i}, i=0, \ldots, n-1$, given by (5) are involutive and constant dimensional on $\mathscr{N}$. Moreover, the distributions $\bar{\Delta}_{i}=\left(\operatorname{span}\left\{\psi_{n-1}, \ldots, \psi_{i+1}\right\}\right)^{\perp}, i=0, \ldots$, $n-2$, and $\bar{\Delta}_{n-1}(x)=T_{x} \mathbb{R}^{n}$ satisfy Theorem 2.2.(b). Application of Theorem 2 completes the proof.

\section{Main result}

The main results of this paper is the following theorem about local state equivalence to the BTF (4) providing a natural, coordinate-free constructive criterion for bijectivity. It turns out that to have such a criterion we have to restrict ourselves to the analytic case. This main result is particularly based on the following Lie algebraic construction (cf. also $[12,13]$ ).

\section{Algorithm 1.}

1. Suppose $g(0) \neq 0$. Put $k_{1}=1, h_{1}=g$ and let $j_{1}$ be such that $g_{j}(0) \neq 0$.

2. For $i>1$ we define $k_{i}$ as the smallest integer such that $\operatorname{ad}_{h_{i-1}}^{k_{i}} f(0)$ is linearly independent of $h_{1}, \ldots, h_{i-1}$ and we put $h_{i}=\mathrm{ad}_{h_{i-1}}^{k_{i}} f$. Finally, $j_{i}$ is defined as the positive integer such that the rows $j_{1}, \ldots, j_{i}$ of the matrix $\left[h_{1}|\ldots| h_{i}\right](0)$ are linearly independent. 
Theorem 3. Consider the analytic system (1). The following statements are equivalent:

1. Locally around the origin the nonlinear system (1) is equivalent to the BTF (2)-(4).

2. Algorithm 1 is applicable to system (1) giving the integers $k_{1}, \ldots, k_{n} ; j_{1}, \ldots, j_{n}$ and vector fields $h_{1}, \ldots, h_{n}$ such that:

(a) $\bar{\Delta}_{i}=\operatorname{span}\left\{h_{1}, \ldots, h_{i+1}\right\}, i=1, \ldots, n-1$, are regular involutive distributions with $\operatorname{dim} \bar{\Delta}_{n-1}=n$. Moreover, these distributions fulfil Theorem 2.2.(b).

(b) For all $i=0, \ldots, n-1$ the $(i+1)$ th order minor $\mu_{i}(x)$ of the matrix $\mathscr{M}_{i}(c f .(6))$ given by the rows $j_{1}, \ldots, j_{i+1}$ is either nonpositive or nonnegative definite on a neighbourhood $\mathscr{N}_{0}$ of the origin.

3. Condition 2 of Theorem $2^{*}$ is valid and for all $i=0, \ldots, n-1$ the following conditions hold (recall that $\Delta_{i}$ 's are given by (5)):

(a) Let $\tilde{\Delta}_{i}$ be an arbitrary distribution having the maximal integral manifold property with

$\Delta_{i}(x) \subset \tilde{\Delta}_{i}(x) \subset\left(\operatorname{span}\left\{\psi_{n-1}, \ldots, \psi_{i+1}\right\}\right)^{\perp} \forall x \in \mathscr{N}_{0}$,

then $\tilde{\Delta}_{i}(x)=\left(\operatorname{span}\left\{\psi_{n-1}, \ldots, \psi_{i+1}\right\}\right)^{\perp} \forall x \in \mathscr{N}_{0}$.

(b) The function $\left\langle\psi_{i}, \mathrm{ad}_{f}^{i} g\right\rangle$ is either nonpositive or nonnegative definite on the neighbourhood $\mathscr{N}_{0}$.

Remark 2. The condition 2.(a) is a straightforward relaxation of a condition considered in [12] for the equivalence to triangular polynomial form of a special type as well as suggested by [13]. Assuming that $k_{1}, \ldots, k_{n}$ are odd, various controllability results were obtained (cf. $[2,16,12]$ ). Let us note that this oddness property is necessary for the validity of 2.(b) of Theorem 3, though, it is not sufficient. The reason is that Algorithm 1 may produce for different systems the same integers $k_{i}$ and vector fields $h_{i}$. Our original contribution here is the computation of integers $j_{1}, \ldots, j_{n}$ to use them in checking 2.(b). Notice, also, that the computation of integers $j_{1}, \ldots, j_{n}$ is, in fact, necessary already for checking the linear independence of $h_{1}, \ldots, h_{n}$.

Remark 3. Theorem 3 is not valid in the smooth case. This is precisely due to the fact that the implication $3 \Rightarrow 2$ does not hold for the general smooth case. Namely, as will be seen during the proof of Theorem 3 , the following statements are valid:

1. Suppose Theorem 3.2 is valid then the smooth system (1) is locally state equivalent to the BTF.

2. Suppose the smooth system (1) is locally state equivalent to the BTF then Theorem 3.3 is valid.
Moreover, both these statements are irreversible, as illustrated by the following counterexamples. First, for the BTF system $\dot{x}_{1}=\phi\left(x_{2}\right), \dot{x}_{1}=u, \phi(x)=$ $\operatorname{sign}(x) \exp (-1 /|x|)$ Theorem 3.2 obviously does not hold. Secondly, consider the smooth function $\psi(x), \psi(0)=0, \psi_{x}(x) \geqslant 0$ such that

$$
\begin{aligned}
& \frac{\partial \psi}{\partial x}(x)= \\
& \begin{cases}\exp \left(\frac{1}{(x-(1 / k))(x-(1 / k+1))}\right) & |x| \in\left(\frac{1}{k+1}, \frac{1}{k}\right), \\
0 & k=1,3,5, \ldots,\end{cases} \\
& \text { elsewhere. }
\end{aligned}
$$

Now, the system $\dot{x}_{1}=x_{2} x_{1}^{2}+\psi\left(x_{2}\right), \dot{x}_{1}=u$ fulfils Theorem 3.3, it is in TF (2)-(3), but it is not in BTF (2)-(4).

Theorem 3.2, although complicated, gives an algorithm of how to check bijectivity in general (possibly nontriangular) coordinates. Theorem 3.3 then provides a geometric, coordinate-free version of Theorem 3.2. Theorem 3 will be proved in the next section. To illustrate it, we give some typical examples where the conditions 2.(a)-(b) are violated.

Example 2. The planar TF system

$\dot{x}_{1}=x_{2}^{2}, \quad \dot{x}_{2}=u$

is clearly not in the BTF and it is not equivalent to BTF since Theorem 3.2.(b) is not valid. Actually, we have

$\mathscr{M}_{2}(x)=\left[\begin{array}{cc}0 & 2 x_{1} \\ 1 & 0\end{array}\right]$

and $\operatorname{det} \mathscr{M}_{2}(x)=-2 x_{1}$. Notice that Theorem 3.2.(a) is valid here, since $\operatorname{ad}_{(0,1)^{\prime}}^{2}\left(x_{2}^{2}, 0\right)^{\prime}=(2,0)^{\prime}$.

Example 3. The planar TF system

$\dot{x}_{1}=x_{1}^{2} x_{2}, \quad \dot{x}_{2}=u$

is not equivalent to BTF since condition 2.(a) of Theorem 3 is not valid. Actually, we have $\operatorname{ad}_{\left(x_{1}^{2} x_{2}, 0\right)^{\prime}}(0,1)^{\prime}=$ $\left(x_{1}^{2}, 0\right)^{\prime}$ and $\operatorname{ad}_{(0,1)^{\prime}} \operatorname{ad}_{\left(x^{2} x_{2}, 0\right)^{\prime}}(0,1)^{\prime}=0$. In terms of Theorem 3.3: the singular distribution $\Delta_{1}$ has the maximal integral manifold property; notice that the onedimensional manifold $x_{1}=0$ is invariant with respect to the system. Both the conditions 2.(b) and 3.(b) of Theorem 3 are valid for this system since

$\mathscr{M}_{0}(x)=\left[\begin{array}{l}0 \\ 1\end{array}\right], \quad \mathscr{M}_{1}(x)=\left[\begin{array}{cc}0 & x_{2}^{2} \\ 1 & 0\end{array}\right]$. 
Remark 4. An important observation following from Theorem 3.3 is that if a given analytic nonlinear system is already in TF and this form is not bijective, then all $T F$ of that system are not bijective. This observation seems to be at first glance obvious, nevertheless, it is not at all clear, how to prove it directly, without using Theorem 3.3. Theorem 3.3 in fact claims that the bijectiveness is coordinate invariant. Actually, by Remark 3, this is valid also in the smooth case.

We conclude this section by giving the following nontrivial (i.e. not being already in the TF) threedimensional example of a system equivalent to the bijective triangular form to illustrate that Theorem 3 really may work in general coordinates.

Example 4. Consider the nonlinear system

$$
\begin{aligned}
& \dot{x}_{1}=x_{2}-2 x_{2} x_{3}^{3}+x_{3}^{2}+4 x_{2} x_{3} u, \\
& \dot{x}_{2}=x_{3}^{3}-2 x_{3} u, \\
& \dot{x}_{3}=u .
\end{aligned}
$$

We have

$g=\left[\begin{array}{c}4 x_{2} x_{3} \\ -2 x_{3} \\ 1\end{array}\right], \operatorname{ad}_{f} g=\left[\begin{array}{c}6 x_{2} x_{3}^{2} \\ -3 x_{3}^{2} \\ 0\end{array}\right], \operatorname{ad}_{f}^{2} g=\left[\begin{array}{c}3 x_{3}^{2} \\ 0 \\ 0\end{array}\right]$

Now, it is an easy exercise to check that the corresponding distributions may be extended to distributions with coristant dimension, namely, the singularity $x_{3}^{2}$ may be factored out for $\Delta_{1}$. Here, the crucial fact is the presence of this singularity also in the first component of ad $f g$. Equivalently, using Theorem 3.3: $k_{1}=k_{3}=1, k_{2}=3 ; j_{1}=1, j_{2}=2, j_{3}=3$ and the corresponding minors are equal to $3 x_{3}^{2}$ and $-9 x_{3}^{4}$, i.e. the system is state equivalent to BTF (2)-(4).

Let us underline that we were able to effectively prove bijectiveness despite the fact that "triangular" coordinates were unknown.

\section{Proof of Theorern 3}

We first state and prove several lemmas necessary for the proof of Theorem 4. The first of them gives necessary and sufficient conditions for the TF (2) to be the BTF (4) in coordinate-dependent form.

Lemma 2. Consider locally around the origin smooth nonlinear sustem (1) and suppose it is already in TF (2)-(3). Then, in a neighbourhood $\mathscr{N}_{0}$ of the origin, this form is BTF (2)-(4) if and only if $g_{n}(0) \neq 0$,

$$
\begin{aligned}
& \forall i=1, \ldots, n-1:\left(\forall x \in \mathscr{N}_{0}, \frac{\partial f_{i}}{\partial x_{i+1}}(x) \geqslant 0\right) \\
& \quad \text { or } \quad\left(\forall x \in \mathscr{N}_{0}, \frac{\partial f_{i}}{\partial x_{i+1}}(x) \leqslant 0\right)
\end{aligned}
$$

and there exists $\varepsilon>0$ such that for all $i=1, \ldots$, $n-1,\left(x_{1}, \ldots, x_{i}\right)^{\prime} \in(-\varepsilon, \varepsilon) \times \cdots(-\varepsilon, \varepsilon)$ :

$$
\begin{aligned}
& \frac{\partial f_{i}}{\partial x_{i+1}}\left(x_{1}, \ldots, x_{i}, x_{i+1}\right) \neq 0 \\
& \quad \text { for almost all } x_{i+1} \in(-\varepsilon, \varepsilon) .
\end{aligned}
$$

Proof. The conditions of the lemma mean that for all $\left(x_{1}, \ldots, x_{n}\right)^{\prime} \in \mathscr{N}_{0}$ and $i=1, \ldots, n-1$ the function $f_{i}\left(x_{1}, \ldots, x_{i}, x_{i+1}\right)$ is strictly monotonous with respect to $x_{i+1} \in(-\varepsilon, \varepsilon)$ since it has almost everywhere nonzero nonpositive (or nonnegative) derivative. The last property is obviously equivalent to (4).

Lemma 3. Consider the smooth nonlinear system (1) in TF (2)-(3). Then the condition (13) of Lemma 2 holds if and only if Theorem 3.3.(b) holds.

Proof. Straightforward.

Lemma 4. Consider the smooth nonlinear system (1) in TF (2-3). If condition (14) of Lemma 2 holds, then condition 3.(a) of Theorem 3 holds.

Proof. Suppose Theorem 3.3.(a) does not hold. This means that there exists $i_{m} \in\{0, \ldots, n-2\}$ such that Theorem 3.3.(a) holds for $i_{m}$ but does not hold for $i_{m}+1$ (recall that $\Delta_{0}$ is always regular at the origin). Particularly, the distribution $\tilde{\Delta}_{i_{n}+1}$ that has the maximal integral manifold property, equals $\Delta_{i_{n}+1}$ on $\mathscr{N}, \overline{\mathcal{N}}=\mathscr{N}_{0}$, and $\operatorname{dim} \tilde{\Delta}_{i_{m}+1}(0)=i_{m}+1(\mathrm{ob}-$ viously, if $\operatorname{dim} \tilde{\Delta}_{i_{m}+1}(0)=i_{m}+2=\operatorname{dim} \Delta_{i_{m}+1}(0)$, then $\tilde{\Delta}_{i_{m}}$ would be regular and Theorem 3.3.(a) would be valid also for $\left.i_{m}+1\right)$. Now, obviously, $\tilde{\Delta}_{i_{m}+1}=\operatorname{span}\left\{\tilde{\Delta}_{i_{m}},\left(\partial f_{n-i_{m}-1} / \partial x_{n-i_{m}}\right)\left(\partial / \partial x_{n-i_{m}}-1\right)\right\}$ with $\tilde{\Delta}_{i_{m}}$ being regular in a neighbourhood of the origin. Therefore $\tilde{\Delta}_{i_{n}+1}$ has on a neighbourhood of the origin in $\mathbb{R}^{n}$ the maximal integral manifold property with $\operatorname{dim} \tilde{A}_{i_{m}+1}(0)=i_{m}+1$ if and only if

$$
\frac{\partial f_{n-i_{m}}-1}{\partial x_{n-i_{m}}}\left(0, \ldots, 0, x_{n-i_{m}}\right) \equiv 0
$$


on a neighbourhood of the origin in $\mathbb{R}$. This contradicts (14) and therefore Theorem 3.3.(a) holds for all $i=$ $0, \ldots, n-1$.

Proof of Theorem 3. $1 \Rightarrow 3$. This is an immediate consequence of Lemmas 2-4. Notice, that this implication is valid also for the smooth case.

$3 \Rightarrow 2$. Theorem 3.3 guarantees, using Theorem $2^{*}$, the existence of triangular coordinates. Straightforward computations show that in the triangular coordinates Theorem 3.3 implies Theorem 3.2. To conclude this part of the proof one has to show that the validity of Theorem 3.2.(a)-(b) in the triangular coordinates implies its validity in all coordinates. This fact is obvious for Theorem 3.2.(a), i.e. particularly we may assume that Algorithm 1 is applicable. Now, let Theorem 3.2.(b) be valid in the triangular coordinates $x$ and let $\tilde{x}=\mathscr{D}(x)$ be arbitrary analytic coordinates. We have $\tilde{\mathscr{M}}_{i}(\tilde{x})=\mathscr{D}_{x}\left(\mathscr{D}^{-1}(\tilde{x})\right) \mathscr{M}_{i}\left(\mathscr{D}^{-1}(\tilde{x})\right)$, where $\tilde{\mathscr{M}}_{i}(\tilde{x})=$ $\left[g(\tilde{x})|\ldots| \operatorname{ad}_{f}^{i-1} g(\tilde{x})\right]$ (see also (6)). Now, apply in the new coordinates $\tilde{x}$ Algorithm 1 to obtain the integers $j_{1}, \ldots, j_{n}$ and vector fields $h_{1}, \ldots, h_{n}$. Since $j_{1}, \ldots, j_{i+1^{-}}$ rows of the matrix $\left[h_{1}(0)|\ldots| h_{i+1}(0)\right]$ are linearly independent and the matrix $\left[h_{1}(x)|\ldots| h_{i+1}(x)\right]$ is lower anti-triangular we have that the minor of $\mathscr{D}_{x}$ given by its last $i+1$ columns and $j_{1}, \ldots, j_{i+1}$-rows is nonzero in a neighbourhood of the origin. As a consequence, sign-definiteness of the minor of $\mathscr{M}_{i}$ given by its $j_{1}, \ldots, j_{i+1}$-rows is not changed during the coordinate change and therefore Theorem 3.2.(b) is valid also in the new coordinates $\tilde{x}$.

$2 \Rightarrow 1$. If Theorem 3.2.(a)-(b) is valid in some coordinates, then by Theorem 2 there obviously exist triangular coordinates and in these coordinates Theorem 3.2.(a)-(b) is valid as well (see the previous part of the proof). Moreover, straightforward computations show that for the TF-system Theorem 3.2 implies (13)-(14) and therefore this TF is the BTF (2)-(4). Notice, that the last part of the proof does not require the analyticity assumption.

\section{Applications to the nonsmooth stabilization}

Here we shortly discuss possible applications of the triangular form to the (nonsmooth) stabilization problem. As was stressed at the beginning, various results dealing with the stabilization of special triangular form systems are available. The following stabilization result is based on [6].
Theorem 4. Consider the smooth nonlinear control system (1) and let Theorem 3.2.(a) be valid with all $k_{1}, \ldots, k_{n}$ being odd. Then this system is locally asymptotically stabilizable using continuous feedback.

Proof. First, note that Theorem 3.2.(a) guarantees that the nonlinear system is state equivalent to the TF and therefore without loss of generality we may assume that our system is already in TF (2)-(3). Now, computing appropriate Lie brackets one can see that the integers $k_{2}, \ldots, k_{n}$ given by Algorithm 1 are such that for all $i=2, \ldots, n$

$\frac{\partial^{k_{i}} f_{n-i-1}}{\partial x_{n-i}^{k_{i}}}(0) \neq 0$

and

$\frac{\partial^{k} f_{n-i-1}}{\partial x_{n-i}^{k}}(0)=0, \quad k<k_{i}$.

Applying [6] - Corollary 1, we obtain that our system is locally asymptotically stabilizable at the origin using continuous feedback.

Finally, let us briefly discuss an application of the BTF to nonsmooth stabilization. In this case, a constructive procedure to find a stabilizing feedback was provided and tested in $[3,5]$. This procedure is, in fact, based on the following proposition.

Proposition 1. Consider the smooth nonlinear system (1) that is state equivalent to the BTF in a neighbourhood $\mathscr{N}_{0}$ of the origin. Then, there exist

1. an open set $\mathscr{N}, \overline{\mathscr{N}}=\mathscr{N}_{0}$,

2. an open set $\mathscr{P}, \bar{P}=\mathscr{P}_{0}, \mathscr{P}_{0}$ being a neighbourhood of the origin in $\mathbb{R}^{n}$,

3. $\mathscr{R}_{0}, \mathscr{S}_{0}-$ neighbourhoods of the origin in $\mathbb{R}$,

4. $\mathscr{D} \in C^{\infty}\left(\mathcal{N}_{0}, \mathscr{P}_{0}\right) \cap \operatorname{DIFF}(\mathcal{N}, \mathscr{P}),{ }^{3} \alpha \in$ $C^{\infty}\left(\mathscr{R}_{0} \times \mathscr{N}_{0}, \mathscr{S}_{0}\right), \forall x \in \mathscr{N}, \alpha(x, \cdot) \in \operatorname{DIFF}\left(\mathscr{R}_{0}, \mathscr{S}_{0}\right)$, such that for any piecewise continuous input $u(t) \in$ $\mathscr{R}_{0} \forall t \in\left[t_{0}, t_{1}\right] \subset \mathbb{R}$ and the corresponding trajectory of $x(t) \in \mathscr{N}_{0} \forall t \in\left[t_{0}, t_{1}\right] \subset \mathbb{R}$ it holds

$\dot{y}_{1}=y_{2}, \quad \dot{y}_{2}=y_{3}, \quad \dot{y}_{n-1}=y_{n}, \ldots, \dot{y}_{n}=v$,

where $y=\left(y_{1}, \ldots, y_{n}\right)^{\mathrm{T}} \in \mathbb{R}^{n}, v \in \mathbb{R}$ and

$y=\mathscr{D}(x), \quad v=\alpha(x, u), \quad x \in \mathscr{N}_{0}, u \in \mathscr{R}_{0}$.

Moreover, let $x$ be the corresponding triangular coordinates, then the set $\mathcal{N}_{0} \backslash \mathcal{N}=\bigcup_{j=1}^{n-1} \mathcal{N}_{j}$ and

\footnotetext{
${ }^{3}$ The set of all diffeomorphisms between $\mathscr{N}$ and $R$.
} 
the sets $\mathcal{N}_{j}=\left\{x \in \mathscr{N}_{0} \mid\left(\partial f_{j} / \partial x_{j+1}\right)(x)=0\right\}, j=$ $1, \ldots, n-1$, are not invariant with respect to the original nonlinear system.

Proof. Suppose without loss of generality that our system is in BTF. To obtain $\mathscr{D}$ and $\alpha$ one may proceed in a standard way:

$y_{1}=x_{1}, \quad y_{2}=f_{1}\left(x_{1}, x_{2}\right)$,

$y_{i}=f_{i-1} \prod_{j=1}^{i-2}\left(\partial f_{j} / \partial x_{j+1}\right)+\beta_{i}(x), i=3, \ldots, n$,

and finally

$v=u g_{n} \prod_{j=1}^{n-1}\left(\partial f_{j} / \partial x_{j+1}\right)+\beta_{n+1}(x)$

Here the $\beta_{i}$ 's stand for some $C^{\infty}$ functions. Now, the claims 1-4 immediately follow from the fact that each of the derivatives $\left(\partial f_{j} / \partial x_{j+1}\right), j=1, \ldots, n-1$, does not change the sign on $\mathscr{N}_{0}$ and is nonzero on $\mathscr{N}$ (using Lemma 2 ).

Finally, suppose $\equiv j=0, \ldots, n-1$ such that $\mathscr{N}_{j}$ is invariant with respect to the BTF-system (2)-(4) and let $\tilde{j}$ be the maximal such $j$. Consider the following auxiliary system,

$\dot{x}_{1}=f_{1}\left(x_{1}, x_{2}\right), \ldots, \dot{x}_{\tilde{j}}=f_{\tilde{j}}\left(x_{1}, \ldots, x_{\tilde{j}+1}\right), \dot{x}_{\tilde{j}+1}=\tilde{u}$,

then this system has $\mathscr{N}_{\tilde{j}}$ as its invariant set. Since $0 \in \mathcal{N}_{\tilde{j}}$, we have that $\left(\partial / \partial x_{\tilde{j}+1}\right) f_{\tilde{j}}\left(0, \ldots, 0, x_{j+1}\right) \equiv 0$ in a neighbourhood of the origin and therefore (14) does not hold. Using Lemma 2 we have that the system in question is not in BTF - i.e. the obvious contradiction.

Remark 5. Despite its lengthy formulation, the idea of the proposition is very simple: consider the original nonlinear system on the manifold $\mathscr{N}$ (with $\overline{\mathscr{N}}=\hat{N}_{0}$ ), then it is equivalent (using a smooth coordinate change and a smooth feedback) to a linear system defined on an open set $\mathscr{P}$ (with closure being again a neighbo urhood of the origin). Moreover, only trajectories produced by very special inputs may lie inside the singular set $\mathscr{N}_{0} \backslash \mathscr{N}$. It can be even shown that in the analytic case only the zero input may produce a trajectory belonging to the singular set.

We illustrate Proposition 1 with the following example.
Example 5. Consider the BTF-system

$\dot{x}_{1}=x_{1}+x_{2}^{3}, \quad \dot{x}_{2}=x_{3}, \quad \dot{x}_{3}=u$.

It is not smoothly stabilizable (it has an unstable approximate linearization) but it is continuously stabilizable using Theorem 4 . Nevertheless, this theorem does not explicitly provide a stabilizing feedback. Using Proposition 1 we have for $y_{1}=x_{1}, y_{2}=x_{1}+x_{2}^{3}, y_{3}=$ $x_{1}+x_{2}^{3}+3 x_{2}^{2} x_{3}, v=x_{1}+x_{2}^{2}+3 x_{2}^{2} x_{3}+6 x_{2} x_{3}^{2}+3 x_{2}^{2} u$ that $\dot{y}_{1}=y_{2}, \dot{y}_{2}=y_{3}, \dot{y}_{3}=v$. Notice that relations between $x$ and $y$ have all properties required by Proposition 1, namely, they provide a diffeomorphism between $\left\{x \in \mathbb{R}^{3} \mid x_{2} \neq 0\right\}$ and $\left\{y \in \mathbb{R}^{3} \mid y_{1} \neq y_{2}\right\}$. An analogous statement is true for $u, v$.

Proposition 1 indicates for a system in bijective triangular form a straightforward approach to its stabilization. Namely, one may easily compute a stabilizing feedback that is not defined at singularities and is unbounded, then this feedback may be "regularized" to obtain a continuous stabilizing feedback. This approach was numerically successfully tested for two- and three-dimensional systems in $[3,5]$.

\section{Conclusions and outlooks}

Necessary and sufficient geometric conditions for the state equivalence to the (bijective) triangular form were provided. Straightforward procedures for their checking in any fixed coordinate system were also provided. Only single-input nonlinear systems were considered. At present it seems to be difficult to give equivalents for these conditions in the multi-input case.

Possible applications for the nonsmooth stabilization were indicated. This direction certainly deserves further investigation.

\section{References}

[1] D. Aeyels, Local and global controllability for nonlinear systems, Systems Control Lett. 5 (1984) 19-26.

[2] D. Aeyels, Stabilization of a class of nonlinear systems by a smooth feedback control, Systems Control Lett. 5 (1985) 289-294.

[3] S. Čelikovský, Topological linearization of nonlinear systems: application to the nonsmooth stabilization, Proc. $2 n d$ ECC'93, Groningen, The Netherlands (1993) 41-44.

[4] S. Čelikovský, Topological equivalence and topological linearization of controlled dynamical systems, Kybernetika 31 (1995) 141-150. 
[5] S. Celikovský, Numerical algorithm for the nonsmooth stabilization of triangular systems Kybernetika 32 (1996), to appear.

[6] J.M. Coron and L. Praly, Adding an integrator for the stabilization problem, Systems Control Lett. 17 (1991) 89-104.

[7] A. Isidori, Nonlinear Control Systems. An Introduction (Springer, Heidelberg, 1989).

[8] B. Jakubczyk and W. Respondek, On linearization of control systems, Bull. Acad. Sci. Polonaise Ser. Sci. Math. 28 (1980) 517-522.

[9] V.I. Korobov, Controllability and stability of certain nonlinear system, Differentsial'nye Uravneniya 9 (1973) 614-619.

[10] H. Nijmeijer and A.J. van der Schaft, Nonlinear Dynamical Control Systems (Springer, New York, 1990).

[11] W. Respondek, On decomposition of nonlinear control systems, Systems Control Lett. 1 (1982) 301-308.

[12] W. Respondek, Global aspects of linearization, equivalence to polynomial forms and decomposition of nonlinear control systems, in: M. Fliess and M. Hazewinkel, eds., Algebraic and Geometric Methods in Nonlinear Control Theory (Reidel, Dordrecht, 1986) 257-283.

[13] W. Respondek, private communication, September 1992.

[14] C. Simões, H. Nijmeijer and J. Tsinias, Nonsmooth stabilizability and feedback linearization of discrete-time nonlinear systems, Memorandum No. 1190, Dept. Applied Mathematics, Univ. Twente, The Netherlands; to appear in: Internat. J. Robust Nonlinear Control.

[15] H. Sussmann, Orbits of families of vector fields and integrability of distributions, Trans. Am. Math. Soc. 180 (1973) 171-188.

[16] H.J. Sussmann, Lie brackets and local controllability: a sufficient condition for scalar input system, SIAMJ. Control Optim. 21 (1983) 686-713.

[17] J. Tsinias, A local stabilization for interconnected systems, Systems Control Lett. 18 (1992) 429-434.

[18] J. Tsinias, Partial-state global stabilization for general triangular systems, Systems Control Lett. 24 (1995) 139-145.

[19] J. Tsinias, Versions of Sontag's input to state stability condition and output feedback global stabilization, to appear. 